\title{
Diacronie
}

Studi di Storia Contemporanea

$\mathrm{N}^{\circ} 31,3$ | 2017

"Le armi della politica, la politica delle armi"

\section{Dallo stomaco al cuore?}

Il rifornimento di cibo per la popolazione civile nella Croazia occupata e i tentativi italiani di costruire un impero

\section{Sanela Schmid}

Traduttore: Alessandro Stoppoloni

\section{CpenEdition}

\section{Journals}

Edizione digitale

URL: http://journals.openedition.org/diacronie/6238

DOI: $10.4000 /$ diacronie. 6238

ISSN: 2038-0925

Editore

Association culturelle Diacronie

Notizia bibliografica digitale

Sanela Schmid, «Dallo stomaco al cuore? », Diacronie [Online], №31, 3 | 2017, documento 5, Messo

online il 29 octobre 2017, consultato il 01 mai 2019. URL : http://journals.openedition.org/

diacronie/6238; DOI : 10.4000/diacronie.6238 


\title{
Diacronie
}

Studi di Storia Contemporanea

$31,3 / 2017$

"Le armi della politica, la politica delle armi". Ideologie di lotta ed esperienze di guerra

\section{Dallo stomaco al cuore? Il rifornimento di cibo per la popolazione civile nella Croazia occupata e i tentativi italiani di costruire un impero}

\author{
Sanela SCHMID \\ Traduzione di Alessandro STOPPOLONI
}

Per citare questo articolo:

SCHMID, Sanela, «Dallo stomaco al cuore? Il rifornimento di cibo per la popolazione civile nella Croazia occupata e i tentativi italiani di costruire un impero», Diacronie. Studi di Storia Contemporanea : "Le armi della politica, la politica delle armi". Ideologie di lotta ed esperienze di guerra, 31, 3/2017, 29/10/2017,

URL: <http://www.studistorici.com/2017/10/29/schmid_numero_31/ >

Diacronie Studi di Storia Contemporanea $\rightarrow$ http://www.diacronie.it

Rivista storica online. Uscita trimestrale.

redazione.diacronie@hotmail.it

Comitato di direzione: Naor Ben-Yehoyada - João Fábio Bertonha - Christopher Denis-Delacour - Maximiliano Fuentes Codera Anders Granås Kjøstvedt - John Paul Newman - Deborah Paci - Niccolò Pianciola - Spyridon Ploumidis - Wilko Graf Von Hardenberg

Comitato di redazione: Jacopo Bassi - Luca Bufarale - Gianluca Canè - Fausto Pietrancosta - Alessandro Salvador - Matteo Tomasoni Diritti: gli articoli di Diacronie. Studi di Storia Contemporanea sono pubblicati sotto licenza Creative Commons 3.0. Possono essere riprodotti e modificati a patto di indicare eventuali modifiche dei contenuti, di riconoscere la paternità dell'opera e di condividerla allo stesso modo. La citazione di estratti è comunque sempre autorizzata, nei limiti previsti dalla legge. 


\title{
5/ Dallo stomaco al cuore? Il rifornimento di cibo per la popolazione civile nella Croazia occupata e i tentativi italiani di costruire un impero
}

\author{
Sanela SCHMID
}

Traduzione di Alessandro STOPPOLONI

Nell'articolo vengono esaminate le strategie di approvvigionamento alimentare poste in essere nel quadro dell'imperialismo italiano nelle regioni annesse e occupate dello Stato indipendente di Croazia. L'approvvigionamento aveva molteplici scopi: la popolazione doveva essere coinvolta in un progetto imperiale, era necessario far sì che gli ustascia fossero screditati di fronte alla popolazione e infine bisognava promuovere il fascismo come assetto futuro e combattere i resistenti.

\footnotetext{
Elenco delle abbreviazioni degli archivi citati

ACS $=$ Archivio Centrale dello Stato

$\mathrm{AJ}=$ Arhiv Jugoslavije

ASMAE $=$ Archivio Storico diplomatico del Ministero degli Affari Esteri

AUSSME $=$ Archivio dell'Ufficio Storico dello Stato Maggiore dell'Esercito

BArch $=$ Bundesarchiv

HDA = Hrvatski Državni Arhiv

NARA = US National Archives and Records Administration

PA AA $=$ Politisches Archiv des Auswärigen Amtes

$\mathrm{VA}=$ Vojni Arhiv
}

L'elemento imperiale dell'Italia fascista sta riscuotendo sempre più interesse fra i ricercatori e in particolare chi si occupa di storia culturale sta lavorando su questo tema con grande profitto ${ }^{1}$. Quando però si sposta l'attenzione sulla politica italiana nelle regioni occupate durante la Seconda guerra mondiale la portata della rappresentazione fascista dell'Impero nei suoi effetti sulle zone di occupazione viene indagata solo in modo superficiale. È innegabile che vi siano libri e articoli che contengono nel titolo il concetto "Impero" e che si dedicano alle occupazioni italiane durante la Seconda guerra mondiale, tuttavia la loro relazione con questo concetto è incentrata

\footnotetext{
${ }^{1}$ BEN-GHIAT, Ruth, Italian fascism's empire cinema, Bloomington, Indiana University Press, 2015.
} 
principalmente sul confronto fra i piani di conquista italiani e le differenze fra la progettata espansione dell'Impero e la sua effettiva realizzazione ${ }^{2}$.

Le idee italiane per un futuro "nuovo ordine" vengono quindi prese poco in considerazione quando si tratta di verificare le effettive politiche di occupazione. Se però si segue lo spunto di Emilio Gentile e si prendono sul serio le implicazioni di miti, simboli e rituali come un «sistema di credenze e valori» $»^{3}$ bisogna anche domandarsi quali conseguenze la propaganda di questi valori per quasi vent'anni abbia avuto sulla politica imperiale italiana durante la guerra. Questo modo di procedere potrebbe aiutare ad affrontare e chiarire meglio la natura bifronte dell'occupazione, un elemento che finora la ricerca ha trascurato o in favore della narrazione del bravo italiano ${ }^{4}$ o di quella dell'esilio dello stesso ${ }^{5}$. Partendo dal caso delle zone occupate e annesse dello Stato indipendente di Croazia (Nezavisna Država Hrvatska, NDH) ci si propone di indagare l'atteggiamento italiano verso il problema dell'approvvigionamento alimentare nel contesto dell'imperialismo fascista. Accanto a fattori politici, diplomatici e militari le rappresentazioni fasciste del tipo di impero hanno determinato le modalità con cui i dominanti italiani, sia civili sia militari, hanno affrontato i problemi. Le idee che hanno informato la politica italiana si nutrivano dell'aspirazione dei fascisti ad essere i successori dell'Impero romano e si basavano sui due pilastri: da una parte la rappresentazione di una posizione di dominio nel Mediterraneo che doveva essere costruita sulla forza militare e dall'altra la convinzione di esprimere una civiltà superiore rispetto ai popoli che vivevano nel proclamato spazio vitale. Entrambi sono stati utilizzati per legittimare l'aspirazione a costruire un impero e allo stesso tempo hanno al contempo costituito un motivo per la creazione di due modi di agire contraddittori. Il punto di

\footnotetext{
2 BURGWYN, James H., Empire on the Adriatic. Mussolini's Conquest of Yugoslava 1941-1943, New York, Enigma Books, 2005; RODOGNO, Davide, Fascism's European Empire: Italian Occupation During the Second World War, Cambridge, Cambridge University Press, 2006 [ed. orig.: Il nuovo ordine mediterraneo. Le politiche dell'Italia fascista in Europa (1940-1943), Torino, Bollati Boringhieri, 2003]; LABANCA, Nicola, L'Impero del fascismo. Lo stato degli studi, in BOTTONI, Riccardo (a cura di), L'impero fascista: Italia ed Etiopia (1935-1941), Bologna, Il Mulino, 2008, pp. 35-61. Per quel che riguarda il tema qui trattato, si veda a p. 37.

3 GENTILE, Emilio, The Sacralization of Politics in Fascist Italy, Cambridge, Cambridge University Press, 1996, pp. 159-160.

4 In italiano nel testo originale così come tutte le espressioni in corsivo che si ritrovano nell'articolo. [N.d.T]. Per la ricerca storica l'immagine del bravo italiano può considerarsi superata. Questo tipo di argomentazione si ritrova soprattutto in testi non recenti e comunque di spessore che si concentrano principalmente su alcuni aspetti dell'occupazione come la politica nei confronti degli ebrei. Cfr. STEINBERG, Jonathan, Deutsche, Italiener und Juden. Der italienische Widerstand gegen den Holocaust, Göttingen, 1992 [ed. orig.: All or Nothing: The Axis and the Holocaust 1941-43, London, Routledge, 1990]; SHELAH, Menachem, Un debito di gratitudine. Storia dei rapporti tra l'Esercito Italiano e gli Ebrei di Dalmazia (1941-1943), Roma, Stato Maggiore dell'Esercito - Ufficio Storico, 1991.

5 I lavori più importanti sulla Jugoslavia sono sicuramente RODOGNO, Davide, Il nuovo ordine mediterraneo, cit.; KNOX, MacGregor, "Das faschistische Italien und die "Endlösung" 1942/43», in Vierteljahrshefte für Zeitgeschichte, 55, 1/2007, pp. 53-92; CONTI, Davide, L'occupazione italiana dei Balcani. Tra crimini di guerra e mito della brava gente, Roma, Odradek, 2008; CACCAMO, Francesco, MONZALI, Luciano (a cura di), L'occupazione italiana della Iugoslavia (1941-1943), Firenze, Le Lettere, 2008. I primi a essersi occupati della dimensione criminale dell'occupazione italiana furono tuttavia COLLOTI, Enzo, SALA, Teodoro (a cura di), Le potenze dell'Asse e la Jugoslavia. Saggi e Documenti 1941/43, Milano, Feltrinelli, 1974.
} 
vista militare ha dato luogo fin dal primo giorno dell'occupazione a una battaglia senza riguardi verso chiunque si opponesse all'espansionismo italiano ed è sfociato nella costruzione di campi di concentramento e in esecuzioni di massa. Allo stesso tempo però gli occupanti italiani promisero a tutti quelli che erano disposti ad aderire all'Impero italiano protezione e un miglioramento nelle condizioni di vita. Cercarono perfino di convincere la popolazione dei vantaggi dell'appartenenza all'Impero fascista. Alcuni degli aspetti della politica di occupazione italiana - la promessa di un trattamento su basi egualitarie e di protezione, di rifornimenti alimentari e di materiale medico così come la propaganda - sono strettamente collegate a questo fattore. Come esempio di questa strategia la lotta senza quartiere a tutti i nemici del progetto imperiale e l'aspirazione a conquistarsi le simpatie del popolo per lo stesso progetto devono essere viste come due facce della stessa medaglia. Le possibilità di scelta e i comportamenti del popolo in questa fase devono al contrario essere tralasciati ${ }^{6}$.

\section{Spazio vitale e impero}

Fin dagli albori del movimento, i fascisti hanno difeso l'idea che la "nuova Italia", un paese moderno e industrializzato, nato dalla prima guerra mondiale dovesse espandersi non solo territorialmente, ma dovesse anche svolgere una missione di civiltà nel mondo moderno ${ }^{7}$. Volevano costruire un nuovo Impero fascista, un proprio spazio vitale. Sebbene questa espressione italiana non sia altro che la traduzione letterale della parola tedesca Lebensraum la rappresentazione italiana del concetto era differente dal suo corrispondente tedesco. Così come il concetto Lebensraum in tedesco nel corso degli anni si è arricchito di nuovi contenuti, anche in italiano ha acquisito un significato proprio. Sotto il nazismo il concetto di Lebensraum si è sviluppato verso la rappresentazione di un struttura razzista nella quale i concetti di razza e classe si intrecciavano fra loro per suddividere intere aree di territorio in base a criteri razzisti. Questo sviluppo è sfociato infine nella politica di deportazione e annientamento portata avanti nel Generalplan-Ost ${ }^{8}$.

\footnotetext{
${ }^{6}$ Questo approccio è stato seguito per esempio da TÖNSMEYER, Tatjana, «Hungerökonomien. Vom Umgang mit der Mangelversorgung im besetzten Europa des Zweiten Weltkriegs» in Historische Zeitschrift, 301, 3/2015, pp. 662-704.

7 GENTILE, Emilio, La nazione del fascismo. Alle origini del declino dello Stato nazionale, in SPADOLINI, Giovanni ( a cura di), Nazione e nazionalità in Italia. Dall'alba del secolo ai nostri giorni, Roma-Bari, Laterza, 1994, pp. 65-124, specialmente alle pp. 68 et seq.; GENTILE, Emilio, La grande Italia, Roma-Bari, Laterza, 1997, p. 172; ZUNINO, Pier Giorgio, L'ideologia del fascismo. Miti, credenze e valori nella stabilizzazione del regime, Bologna, Il Mulino, 1985, pp. 356-358. Questa idea si affermò in modo particolare dopo la conquista dell'Etiopia.

8 JUREIT, Ulrike, Das Ordnen von Räumen. Territorium und Lebensraum im 19. und 20. Jahrhundert, Hamburg, Hamburger Edition, 2012, in particolare alle pp. 287-386; RÖSSLER, Mechthild, SCHLEIERMACHER, Sabine (herausgegeben von), Der "Generalplan Ost". Hauptlinien der nationalsozialistischen Planungs- und Vernichtungspolitik, Berlin, Akademie Verlag, 1993. Il „Generalplan Ost“ si trova online, URL:

<http://www.1000dokumente.de/index.html?c=dokument_de\&dokument=0138_gpo\&object=translation\&st $=\& \mathrm{l}=\mathrm{de}>$ [consultato il 14 ottobre 2017].
} 
I piani fascisti per la creazione dell'Impero dovevano essere idealmente realizzati nei grandi spazi africani, ma trovarono applicazione essenzialmente in piccoli Stati e nazioni all'interno dello spazio imperiale. Si procedette quindi a creare una struttura con una grande potenza (l'Italia) al centro che avrebbe dettato i principi per la nuova civiltà. Allo stesso tempo ebbe anche un ruolo la rappresentazione di una missione civilizzatrice per cercare di rendere evidenti ai popoli occupati i vantaggi di una conquista fascista9. L'idea fascista era quindi molto più vicina all'imperialismo inglese e francese del diciannovesimo secolo che alla politica di espansione nazista che prevedeva la conquista di grandi spazi da mettere a disposizione della popolazione tedesca $^{10}$.

La rappresentazione degli eredi ideali di Roma, la romanita ${ }^{11}$, era una costruzione ideologica molto elastica e ha rappresentato uno dei pilastri dell'identità fascista dall'inizio del movimento fino alla sua fine e durante il ventennio era onnipresente ${ }^{12}$.

Essa rappresentava un insieme ideologico abbastanza coerente che sosteneva sia il concetto di Stato totalitario fascista sia l'imperialismo che mirava a diffondere le "virtù romane", così come venivano intese dai fascisti, nel mondo. L'accesso unico alla civiltà romana presupponeva la presenza di Diritto, Ordine e Giustizia come principi base della romanità ${ }^{13}$. Dopo la conquista dell'Etiopia e la proclamazione dell'Impero divenne pratica comune, tanto nella cultura "alta" come in quella più popolare, il richiamo alla storia romana per giustificare la politica fascista ${ }^{14}$. La definizione dell'Impero rimase sempre vaga, ma venne tuttavia sempre collegata a un lato spirituale. Proprio l'inesattezza e la flessibilità del dibattito sul concetto di impero fascista ha permesso il suo utilizzo nella propaganda in modo vario. Così durante l'intero ventennio rimase costante una dicotomia fra la rappresentazione militare e quella conciliante dell'Impero ${ }^{15}$. Questo risulta particolarmente importante per la ricerca sugli effetti dei concetti imperiali fascisti

9 GENTILE, Emilio, La Grande Italia, cit., pp. 172-182.

${ }^{10} \mathrm{Cfr}$. RODOGNO, Davide, Sogni di conquista e realtà delle occupazioni fasciste in Europa, 1940-1943, in GRIBAUDI, Gabriella (a cura di), Le guerre del Novecento, Napoli-Roma, L'Ancora del Mediterraneo, 2007, pp. 113-121, pp. 116 et seq.

${ }^{11}$ Per una dettagliata analisi del concetto di romanità sotto il Fascismo si rinvia a NELIS, Jan, From ancient to modern: The myth of romanità during the ventennio fascista. The written imprint of Mussolini's cult of the "Third Rome", Bruxelles-Roma, Institut Historique belge de Rome, 2011, in particolare le pp. 36, 169. Si veda anche la voce «Romanità» in De GRAZIA, Victoria, LUZZATTO, Sergio (a cura di), Dizionario del fascismo, Vol. 2, Torino, Einaudi, 2003, pp. 539-541.

12 STONE, Marla, A flexible Rome: Fascism and the cult of romanità, in EDWARDS, Catharine (ed.), Roman Presences: Receptions of Rome in European Culture, 1789-1945, Cambridge-New York, Cambridge University Press, 1999, pp. 205-220, p. 205; NELIS, Jan, From ancient to modern, cit., pp. 40, 171.

13 VISSER, Romke, «Fascist Doctrine and the Cult of the Romanità», in Journal of Contemporary History, 27, 1/1992, pp. 5-22, in particolare pp. 15-17.

14 GENTILE, Fascismo di pietra, Roma-Bari, Laterza, 2007, p. 206; STONE, Marla, A Flexible Rome, cit., pp. 208219.

15 NELIS, Jan, «Imperialismo e mito della romanità nella Terza Roma Mussoliniana», in Forum Romanum Belgicum, 2012, p. 7, URL: < http://kadoc.kuleuven.be/bhir-ihbr/doc/3_forum_nelis.pdf > [consultato il 27 febbraio 2017]. 
rispetto a quanto veniva poi fatto sul posto perché proprio questi due elementi contraddittori hanno determinato la politica di occupazione, come verrà mostrato in seguito, usando come esempio l'approvvigionamento di beni alimentari.

\section{Occupazione e situazione alimentare in NDH}

Dopo lo smembramento della Jugoslavia ad opera delle potenze dell'Asse nell'aprile del 1941, venne creato lo Stato indipendente di Croazia alla cui testa venne posto il movimento fascista ustascia che doveva farsi garante di una politica favorevole all'Asse all'interno del paese ${ }^{16}$. Allo stesso tempo i due alleati divisero il paese secondo sfere di influenza: alla Germania spettò il Nord mentre l'Italia ottenne il Sud. Il Reich tedesco si accontentò di difendere i suoi interessi economici e di esercitare, attraverso i suoi rappresentanti diplomatici e militari a Zagabria, un'influenza sul regime ustascia ${ }^{17}$. L'Italia al contrario aveva dei forti interessi espansionistici sulla Croazia e la collaborazione con gli Ustascia, in precedenza a lungo protetti da Mussolini, rendeva chiaramente più difficile raggiungere questi obiettivi ${ }^{18}$. Con i protocolli di Roma del 18 maggio 1941 venne sancita definitivamente l'annessione della maggior parte della Dalmazia all'Italia, anche se la parte più interna rimaneva alla Croazia. Questa decisione dispiacque in modo particolare al generale Vittorio Ambrosio, il comandante della 2a Armata italiana che si trovava in Slovenia e Croazia, che da un punto di vista militare avrebbe preferito stabilire il confine lungo la cresta delle Alpi dinariche ${ }^{19}$. Nel settembre del 1941 le truppe italiane occuparono il territorio corrispondente alla loro sfera di influenza in Croazia e divisero quest'area in due zone. Mentre nella seconda zona, collegata direttamente alla costa dalmata e denominata di solito dalle truppe di occupazione prima zona, la violenza militare e civile fu esercitata direttamente dalle truppe d'occupazione italiane, nella terza l'esercito italiano si limitò a un'attività di controllo. Nell'agosto del 1942 quest'ultima zona venne evacuata dagli italiani che lasciarono nell'altra solo delle teste di ponte. Soprattutto durante il primo anno di occupazione, ma anche successivamente, il generale Ambrosio e il suo successore si preoccuparono di assicurarsi militarmente quello che non era stato raggiunto tramite una via diplomatica. Si comportarono così come capomastri

\footnotetext{
${ }^{16}$ HORY, Ladislaus, BROSZAT, Martin, Der kroatische Ustascha-Staat, 1941-1945, Stuttgart, Deutsche VerlagsAnstalt, 1964.

17 SUNDHAUSSEN, Holm, Wirtschaftsgeschichte Kroatiens im nationalsozialistischen Großraum 1941-1945. Das Scheitern einer Ausbeutungsstrategie, Stuttgart, Deutsche Verlags-Anstalt, 1983.

18 GOBETTI, Eric, Dittatore per caso. Un piccolo duce protetto dall'Italia fascista, Napoli, L'Ancora del Mediterraneo, 2001; SADKOVICH, James, Italian Support for Croatian Separatism 1927-1937, New York, Garland, 1987.

19 Bundesarchiv (Archivio di stato tedesco, BArch), RH 31-III/8, Nr. 18, Bericht der deutschen Gesandtschaft an das Auswärtige Amt, 31.12.1941.
} 
dell'Impero italiano, prodigandosi per un allargamento sia della zona italiana sia della sfera d'influenza.

Vennero quindi determinati degli obiettivi diplomatici, degli obblighi militari e un'idea di impero oltre alle modalità con cui i comandanti della 2a Armata così come le loro divisioni avrebbero governato le regioni occupate. Questo viene mostrato chiaramente già nel comunicato del 7 settembre 1941 con il quale l'esercito italiano, dopo aver preso il controllo delle regioni croate, annunciò fra le altre cose la fine delle persecuzioni e una nuova politica di non discriminazione. Di conseguenza, in forte contrasto con la politica vigente fino a quel momento in Croazia e rivolta contro i suoi abitanti serbi, ebrei e rom: tutte le persone che avevano lasciato i loro paesi furono invitate a tornare indietro con la promessa che le loro vite, la loro libertà e le loro proprietà sarebbero state protette ${ }^{20}$.

Innumerevole materiale propagandistico venne distribuito fra la popolazione per convincerla dei vantaggi derivanti dall'appartenenza all'impero italiano. Per fare ciò la propaganda utilizzò soprattutto i concetti di latinità, giustizia romana e civiltà romana. Un rapporto dell'ottobre 1941 sulla situazione in una località della seconda zona mostra come gli italiani volessero essere visti: «[...] la presenza dei nostri soldati dà motivo a schiette manifestazioni di simpatia verso l'Italia di cui si ammira particolarmente il senso di umana giustizia dovuto ad una civiltà superiore» ${ }^{21}$. Allo stesso tempo la lotta contro chi si opponeva all'occupazione diffuse paura e spavento all'interno della popolazione che fu vittima di una spirale di violenza comprendente aggressioni, controaggressioni, e soprattutto rastrellamenti che portarono essenzialmente a imprigionamenti, esecuzioni e saccheggi ${ }^{22}$.

I comandanti della 2a Armata di fronte alla popolazione cercarono di minimizzare gli eccessi che con i loro stessi ordini avevano provocato. Tali "contrordini" si basavano sempre sulla sottolineatura della superiore civilizzazione italiana ${ }^{23}$. Essi però dimostravano di non essere in grado di mantenere la disciplina nell'esercito, cosicché durante i rastrellamenti i saccheggi rimanevano all'ordine del giorno ${ }^{24}$.

\footnotetext{
20 Archivio dell'Ufficio storico dello Stato maggiore dell'esercito (AUSSME), N. 1-11 Diari storici seconda guerra mondiale (DS) 583, b. 154, no. 3, Proclama alla popolazione, 7 settembre, 1941.

${ }^{21}$ Ibidem, b. 543, All. 167 al DS di ottobre 1941, Comando della divisione fanteria "RE", Uff. Affari civili, Relazione, 15 ottobre 1941.

22 Vojni Arhiv (Archivio militare, VA), TA, k. 91, reg. br. 2/4-6, Operazioni contro i ribelli, 13.1.1942; LEGNANI, Massimo (a cura di), «Il "ginger" del generale Roatta Le direttive della 2a Armata sulla repressione antipartigiana in Slovenia e Croazia», in Italia Contemporanea, 209-210, 1997-1998, pp. 155-174, pp. 161 et seq.

23 VA, TA, k. 694, reg. br. 29/2, Danneggiamenti, 21.7.1943.

24 Ci limitiamo a citare una piccola selezione dei documenti sull'argomento: AUSSME, DS, b. 646, All. 294, al DS, Rapporto situazione per il mese di Aprile 1942; AUSSME, DS, b. 1426, All. 67, al DS, Comando V CdA, Controguerriglia e distruzione di case, 6.6.1942; AUSSME, DS, b. 782, All. 235, al DS, Relazione sull'operazione di Velebit, 2.8.42; AUSSME, DS, b. 646, All. 5, al DS Marzo 1942, Telescritto XVIII CdA al Comando 2. Armata; HDA, OUP, k. 41, Nr. 16509, Mostar, 4.5.1943; HDA, OUP, k. 24, Nr. 14795, Rapporto dal
} 
Questo era disastroso dal momento che un problema molto grande, non solo per lo Stato croato ma anche per le potenze occupanti, era garantire il sostentamento della popolazione. Questo compito in realtà sarebbe spettato allo Stato croato, ma l'amministrazione non era in grado di garantire l'approvvigionamento alimentare alle regioni meno produttive dal punto di vista agricolo come l'Erzegovina, l'entroterra dalmata, le isole, così come alcune regioni della Bosnia. Queste regioni, eccezion fatta per la Dalmazia, che era sotto la responsabilità italiana, dovevano essere rifornite di alimenti come grano, mais e zucchero dallo Stato jugoslavo anche in tempo di pace. La 2a Armata aveva sostenuto già nel maggio del 1941 che il problema dell'alimentazione era uno dei più seri che il governo croato doveva risolvere ${ }^{25}$.

Le autorità italiane e tedesche fin dall'inizio riferivano di una situazione alimentare critica che inoltre continuava a peggiorare. Già fra la fine di aprile e l'inizio di maggio del 1941 le divisioni italiane resero noto che la situazione dei rifornimenti sulla costa e nell'entroterra era critica e che diventava sempre più difficile. Sulle isole si era già in carestia e in alcune località dell'Erzegovina si erano verificate delle morti per fame. Nell'estate del 1941 non c'era più pane, carne o verdure; nei negozi si creavano delle file e i prezzi salivano ${ }^{26}$.

La cattiva situazione alimentare aveva diverse cause. In primis la Croazia non era ricca di prodotti agrari, al contrario di quanto pensavano alcuni amministratori tedeschi. A fronte di una produzione eccedente in Slavonia, c'erano altre regioni che non riuscivano a coprire il loro fabbisogno. Questa situazione peggiorò drammaticamente quando, in seguito allo spezzettamento e alla spartizione della Jugoslavia, altre regioni con una forte produzione agricola come la Voivodina vennero completamente separate dal mercato croato. A ciò si aggiunse il cattivo raccolto del 1941, in cui la produzione di grano ammontò a meno di un terzo rispetto alle quantità abituali ${ }^{27}$. Secondo quanto affermato dai croati la produttività agricola fu il $50 \%$ di quella media fatta registrare fra il 1937 e il 1939. L'inverno 1941-1942 fu inoltre particolarmente lungo e in Slavonia si registrarono anche delle inondazioni. In aggiunta la bassa qualità delle sementi lasciava anche poche speranze per l'anno seguente ${ }^{28}$.

Un secondo motivo era la presenza di entrambi gli eserciti di occupazione che riduceva la quantità di cibo disponibile per la popolazione ${ }^{29}$. Inoltre gli eserciti non rispettarono gli accordi

Kotarska Oblast Gračac, Sušak, 2.9.1942.

25 NAW, T-821, R. 278, Nr. 423-430, Notiziario Politico Militare, 17.05.1941.

${ }^{26}$ Archivio storico del Ministero degli Affari Esteri (ASMAE), Gabinetto armistizio-pace (GAB-AP) 1923-1943, b. 1493, Rapporto dall'Ispettore Generale di P.S., Zagabria, 17.6.1941.

27 BArch, RH 31-III/5, Bericht „Die Volksernährung in Kroatien“ vom Chef der kroatischen Militärpropaganda, Donegani, Zagreb, 4.10.1942.

28 SUNDHAUSSEN, Holm, op. cit., p. 265; PA-AA, Gesandtschaft Zagreb, Geheimakten, P. 2, Anlage zum Bericht vom 12. Mai 1942, Kult LA-846/42.

29 Protokoll der Sitzung vom 19.1.1942, in JAREB, Jere, Državno gospodarstveno povjereništvo Nezavisne Države Hrvatske od kolovoza 1941. do travnja 1945. godine. Dokumentarni prikaz, Zagreb, Hrvatski Institut za povijest, 2001 , pp. 136 et seq. 
che prevedevano la loro autonomia in merito all'alimentazione dei soldati e comprarono alimenti sul mercato croato, pur essendo coscienti di pagare un prezzo molto più alto di quello stabilito dallo Stato croato. I soldati italiani ricevevano denaro per comprare gli alimenti di cui avevano bisogno e di conseguenza i contadini non avevano più bisogno di portare i loro prodotti al mercato visto che potevano venderli direttamente all'esercito ${ }^{30}$.

A ciò si aggiunse l'esportazione illegale operata dall'esercito italiano di alimenti e bestiame verso le regioni annesse all'Italia (la Dalmazia) allo stesso modo in cui all'inizio dell'occupazione la Wehrmacht aveva a sua volta esportato illegalmente bestiame in Serbia ${ }^{31}$.

Un terzo motivo erano la guerra e le persecuzioni degli ustascia che creavano degli enormi danni all'agricoltura. Una parte della popolazione fu assassinata o scappò, un'altra venne rinchiusa nei campi di concentramento croati, tedeschi e italiani. In aggiunta a ciò venne operato un trasferimento della forza lavoro verso la Germania per coltivare i campi e per occuparsi della raccolta. I croati che si erano installati sui campi dei serbi cacciati o uccisi non sapevano necessariamente come coltivarli ${ }^{32}$. La pessima situazione alimentare fece sì che anche le sementi venissero usate come cibo e per questo motivo all'inizio del 1942 venne a mancare il 75\% dei semi di frumento. In totale quasi un terzo delle superficie agricola rimase incolta ${ }^{33}$. Quarto, la rivolta aggravò la scarsità di alimenti. L'esercito dei partigiani doveva essere nutrito attraverso consegne spontanee da parte della popolazione e, nel caso in cui queste non fossero bastate, tramite delle requisizioni ${ }^{34}$. Allo stesso tempo distruggevano i raccolti per non farli cadere nelle mani degli occupanti ed esortavano i contadini a produrre solo per i loro fabbisogni ${ }^{35}$.

Infine essi distruggevano le vie di comunicazione così che gli alimenti, anche qualora fossero stati disponibili, non avrebbero potuto essere portati ai centri di distribuzione ${ }^{36}$. D'altra parte gli

\footnotetext{
$3^{30}$ Hrvatski Državni Arhiv [Archivio di Stato Croato, HDA], Opće upravno povjereništvo. [Commissariato Generale Amministrativo Croato, OUP], k. 12, Nr. 13285, Autorità locali di Dubrovnik al Commissariato Civile Croato, 17.2.1942; HDA, OUP, k. 18, Nr. 13974, Autorità locali di Prozor al Commissariato Civile Croato, 17.5.1942; HDA, OUP, k. 2, Nr. 11854, Snabdijevanje Talijanske vojske hranom, 18.9.1941.

${ }^{31}$ SUNDHAUSSEN, Holm, op. cit., p. 267, in particolare la nota 238.

32 Politisches Archiv des Auswärtigen Amtes [Archivio Politico del Ministero degli Affari Esteri Tedesco,PAAA], Gesandtschaft Zagreb, Geheimakten, P. 2, D kult 3 - A 167/42 - Ang.II, Propagandatätigkeit in den Monaten März und April 1942.

33 SUNDHAUSSEN, Holm, op. cit., p. 266.

34 Zbornik, IX/3, Nr. 193, Uputstvo Štaba 12. krajiške brigade 4. krajiške NOU divizije od 14. juna 1943. štabovima bataljona o političkom radu i pravilnom postupku prilikom pribavljanja materijalnih sredstava od naroda, pp. 813-815; Per furto ai campi dei contadini i partigiani arrestati venivano puniti con la morte e la loro condanna veniva resa nota alla popolazione. Arhiv Jugoslavije (Archivio della Jugoslavia, AJ), Fond 110, Inv. Br. 15224, Nr. 597-639, Oglas.

35 BArch, RH 21_2/754, pag. 82, Aufruf zur Ernte durch Partisanen.

36 TALPO, Oddone, Dalmazia. Una cronaca per la storia, vol. 3, Roma, Ufficio Storico Stato Maggiore dell'Esercito, 1994, Situazione alimentare in Croazia, 24.4.1943, pp. 553-555.
} 
stessi occupanti finivano per distruggere $\mathrm{i}$ raccolti come danno collaterale della lotta ai partigiani ${ }^{37}$.

Quinto, il mercato nero, la corruzione e le prevaricazioni operate da determinati gruppi privilegiati come gli Ustascia peggiorarono ulteriormente la situazione. I contadini rifiutavano di vendere il poco che riuscivano a ottenere dai loro campi per un prezzo ridicolo sul mercato legale e preferivano vendere i loro beni sul mercato nero a un prezzo decisamente più elevato ${ }^{38}$. Oppure venne loro impedito di raggiungere i centri abitati più grandi e di praticare il commercio. L'inflazione aumentò senza sosta e nemmeno i tentativi di introdurre dei prezzi calmierati per i beni di prima necessità riuscirono a cambiare molto, soprattutto quando gli alimenti non erano proprio più disponibili ${ }^{39}$. Già nel maggio del 1941 gli operai della strada di Makarska chiesero di essere pagati in farina piuttosto che in denaro, con il quale ormai non si poteva comprare più nulla $a^{40}$. Fino al 1943 la kuna, la moneta croata, valeva così poco che persino la Wehrmacht offriva ricompense sotto forme di farina, zucchero, sale e, in caso di necessità, anche abiti oltre al denaro per chi rivelava i nascondigli di armi ${ }^{41}$.

\section{L'Italia e la questione dei rifornimenti}

Il problema dei rifornimenti e dell'alimentazione non aveva la stessa importanza per la Germania o per l'Italia. Ciò dipendeva da due fattori. Il primo era che la maggioranza delle aree che si trovavano a soffrire di una scarsezza alimentare si trovavano sotto dominio italiano. Secondo, le due potenze occupanti avevano in Croazia differenti obiettivi da raggiungere. La Germania si preoccupava di farsi coinvolgere il meno possibile nelle questioni croate. Il problema dell'alimentazione venne trascurato e si intervenne solo dietro richiesta ufficiale del governo croato. Così per esempio il ministro degli esteri croato nell'estate del 1941 chiese alla Wehrmacht di ottenere alimenti dai loro magazzini occupati per cercare di far fronte alla situazione catastrofica in Bosnia ${ }^{42}$.

37 HDA, OUP, k. 17, Nr. 18906, OUP Komandi 2. Armije, Sušak, 24./25 4.1942; LEPRE, George, Himmler's Bosnian Division. The Waffen-SS Handschar Division 1943-1945, Atglen, Schiffer Military History, 1997, pp. 1174 et seq.

${ }^{8} 8$ BArch, RH 31-III/5, „Die Volksernährung in Kroatien; BArch, RH 31-III/9, Aktenvermerk: Zur Serbenfrage im kroat. Raume, 1.9.1942.

39 VA, NDH, k. 69, reg. br. 3/17-7, Zapovjedničtvo II. Domobranskog zbora, doglasno izvješće 1.-16.2.1942.

40 AUSSME, DS, b. 580, b. 33, Nr. 9, Comando VI CdA, Notiziario Nr. 5, 5.5.1941.

41 BArch, RH 26-114/26, Überwachung der Zivilbevölkerung, 10.10.1943. All'inizio del 1943 cento kune valevano ufficialmente trentotto lire, ma le banche di Spalato pagavano solo diciassette lire. In questo periodo per esempio un litro d'olio costava quattrocentosessanta kune (centosettantacinque lire), sempre che fosse disponibile sul mercato. AUSSME, DS, b. 1068, All. 205, al DS feb. 1943, Relazione mensile sul servizio „A“ 15.1. - 15.2.1943.

42 PA-AA, Gesandtschaft Zagreb, Akten, P. 98, W4 - 368/41, Verbalnote, Zagreb, 9.6.1941. Il Reich però non esaudì la richiesta. 
Al contrario l'Italia si trovava ad agire nel suo spazio vitale. Doveva dimostrare di essere in grado di provvedere ai fabbisogni della popolazione nella Dalmazia annessa se voleva legittimare il suo dominio nel lungo periodo. Nel resto delle zone occupate dall'Italia gli italiani cercarono di sostituirsi allo Stato croato sulla questione degli approvvigionamenti. Da una parte il potere delle autorità doveva essere limitato il più possibile e dall'altra bisognava mostrare al popolo che un dominio italiano doveva essere preferibile a quello degli Ustascia. Subito dopo l'occupazione gli italiani iniziarono a distribuire alimenti ${ }^{43}$. Per fare fronte alla situazione si pensò di introdurre un razionamento del pane. I soldati dovevano astenersi dal comprare pane nei negozi per non aggravare le mancanze ${ }^{44}$. Sebbene il Ministero della guerra italiano nell'aprile del 1941 avesse ordinato che per i bisogni della popolazione i commissari civili dovessero rivolgersi ai ministeri competenti e che l'esercito non dovesse mettere nulla a disposizione i comandanti italiani non rispettarono il divieto. Il comandante della sesta armata, il generale Renzo Dalmazzo, aveva già stabilito in questo momento che "il problema [era] importante, serio e pressante" sia per gli effetti politici sia perché il tema poteva essere efficacemente usato come propaganda contro le truppe italiane ${ }^{45}$. Fino alla metà di maggio 1941 le armate che stazionavano in Croazia e Dalmazia avevano distribuito 62 tonnellate di alimenti alla popolazione e gliene avevano vendute altre 521,5 tonnellate $^{46}$. Dopo diverse vendite di farina la quinta armata, stazionante nella seconda zona si rese conto che una rapida soluzione del problema alimentare rappresentava un ottimo strumento per influenzare la popolazione, in particolare quella più povera ${ }^{47}$. Questo strumento sarebbe stato usato anche successivamente. Allo stesso tempo venne detto con insistenza ai soldati di non provocare danni alle colture; dato che la maggioranza della popolazione faceva il contadino la cattiva reputazione dell'esercito avrebbe messo a repentaglio anche gli obiettivi della guerra $^{48}$.

\footnotetext{
43 DIKULIĆ, Marjan, «Vrsi pod talijanskom okupacijom u Drugom svjetskom ratu», in Radovi Zavoda za povijesne znanosti HAZU u Zadru, 49, 2007, pp. 721-783, p. 728; BARBARIĆ, Juraj, Talijanska politika u Dalmaciji 1941. godine, Split, Institut za historiju radničkog pokreta Dalmacije, 1972, pp. 154 et seq.

44 AUSSME, DS; b. 580, b. 35, Nr. 5, Comando VI CdA, Foglio d'ordini, 7.5.1941; US National Archives and Records Administration (NARA), T-821, R. 278, Nr. 431-440, Notiziario Politico Militare, 31.5.1941. Simili rapporti arrivarono dal commissario civile per la Dalmazia così come da singoli comandanti di divisione. 45 AUSSME, DS, b. 580, rac. 33, Nr. 9, Comando VI CdA, Notiziario Nr. 5, 5.5.1941.

46 AUSSME, DS, b. 726, All. 506, Ministero della Guerra - Gabinetto, 23.4.1941; AUSSME, DS, b. 726, All. 548, Stato Maggiore, Cessione di derrate alle popolazioni locale, 13.5.1941.

47 AUSSME, DS, b. 332, All. 16, Notiziario n. 4, giugno 1941.

48 AUSSME, DS, b. 332, All. 12, Riordinamento ed addestramento dei reparti - circolare 10 U.C. - SMRE, Danni alle coltivazioni, 4.6.1941.
} 


\section{Dissidi fra Italia e Croazia}

Per garantire i rifornimenti alla popolazione civile della Dalmazia il ministero dell'agricoltura italiano concluse un contratto con la società statale croata Pogod. La società si impegnava a consegnare ulteriormente cibo alla Dalmazia. La quantità che doveva essere settimanalmente consegnata consisteva in trenta-quaranta vagoni 49.

La situazione dei rifornimenti alimentari peggiorò dopo l'annessione della Dalmazia. Ai motivi economici si sommarono i giochi di potere. Per gli Ustascia l'annessione della Dalmazia era un'amara sconfitta che costituiva un forte fattore di discredito nei confronti della popolazione ${ }^{50}$. Di conseguenza essi fecero di tutto per ostacolare il consolidamento del dominio italiano e il problema dei rifornimenti offrì loro una gradita opportunità. Per dimostrare la dipendenza della Dalmazia dall'entroterra croato le autorità croate si preoccuparono che nessun bene passasse la frontiera. I militari italiani dedussero che anche attraverso la scarsità di approvigionamenti alimentari avrebbero potuto alimentare il malcontento della popolazione nei confronti dell'amministrazione italiana in Dalmazia51. A luglio il governatore della Dalmazia Giuseppe Bastianini si lamentò di ciò con il Ministro degli Esteri italiano e chiese una soluzione rapida al problema sempre più grave dei rifornimenti. Secondo Bastianini per mantenere il suo prestigio e per giustificare le sue aspirazioni l'Italia doveva dimostrare di essere in grado di provvedere ai bisogni della Dalmazia senza ricorrere alle risorse dell'entroterra. Propose quindi di inviare in Dalmazia ogni tipo di alimenti visto che doveva essere trattata come se fosse un'altra isola ${ }^{52}$. Dato che Luca Pietromarchi del Ministero degli Esteri gli venne incontro venne stilata una lista comprendente gli alimenti di cui la Dalmazia aveva bisogno. Da queste ricerche si capì che la Dalmazia poteva autonomamente coprire circa il $35 \%$ del suo fabbisogno di frumento e l'intero fabbisogno di olio. Tutti le altre derrate alimentari dovevano essere importati. Molti altri alimenti e beni di consumo quotidiano vennero inclusi nel «piccolo commercio di confine» con la Croazia che poteva essere realizzato senza permessi e senza dover pagare delle tasse 53 . I croati tornarono a dirsi d'accordo sul fatto che la Pogod rifornisse la Dalmazia con i principali beni di consumo. L'unica condizione era che i beni venissero suddivisi equamente fra la popolazione italiana e quella croata 54 .

\footnotetext{
49 ASMAE, GAB-AP 1923-1943, b. 1512, Protocollo dell'accordo relativo all'approvvigionamento della Dalmazia da parte della società "Pogod", Zagabria, 12.5.1941.

50 RAMET, Sabrina P., Die drei Jugoslawien. Eine Geschichte der Staatsbildungen und ihrer Probleme, München, De Gruyter Oldenbourg, 2011, p. 184.

${ }^{11}$ AUSSME, DS, b. 449, All. 14, Notiziario Nr. 19, 2.8.1941.

52 ASMAE, GAB-AP 1923-1943, b. 1512, Lettera di Giuseppe Bastianini a Luca Pietromarchi, Roma, 10.7.1941.

53 ASMAE, GAB-AP 1923-1943, b. 1512, Lettera di Bastianini a Pietromarchi, Zara, 14.7.1941 e tre allegati.

54 ASMAE, GAB-AP 1923-1943, b. 1512, Appunto per la R. Legazione, Zagabria, 15.7.1941.
} 
Anche dopo questo accordo, tuttavia, la situazione non migliorò dal momento che l'amministrazione croata perseverava nella sua politica di ostruzionismo, che era sì rivolta contro gli italiani, ma non trascurava le etnie perseguitate nella NDH. Il Ministro croato per l'Industria e il Commercio spiegò all'inviato italiano a Zagabria che non potevano essere inviati alimenti verso Spalato per nutrire gli ebrei e i serbi che si erano rifugiati lì5s. Il mutamento avvenne gradualmente attraverso un accordo sottoscritto al momento della presa di potere da parte dell'esercito italiano nella seconda zona nel settembre del 1941; successivamente, nell'ottobre dello stesso anno si giunse al superamento della frontiera doganale fra la Dalmazia e la seconda zona $^{56}$. Il governo croato si impegnò a subentrare nel garantire i rifornimenti per la popolazione civile e per l'esercito nelle zone d'operazione militari, la seconda e la terza zona, cioè nel loro stesso Stato. Se però il governo non si fosse dimostrato in grado di rispettare l'impegno, i militari avrebbero dovuto sostituirlo e accordarsi per un indennizzo con gli organi amministrativi croati ad esso preposti. L'approvvigionamento della Dalmazia, così come per tutte le altre province, venne assegnato alla sezione "nutrizione" del Ministero per l'Agricoltura e delle Foreste che faceva arrivare alle tre province gli alimenti necessari57. Sebbene l'approvvigionamento della Dalmazia fosse difficile l'amministrazione italiana riuscì a provvedere in modo abbastanza efficace al nutrimento della popolazione ${ }^{58}$. Ciò fu riconosciuto anche da David Sinčić, commissario generale croato presso la 2a Armata italiana, nella primavera del 1943. Egli esortò anche a emulare i procedimenti italiani nelle province confinanti per dimostrare alla popolazione che lo Stato croato era in grado di provvedere ai suoi bisogni 59 .

Anche nella seconda e nella terza zona le autorità italiane e croate litigavano senza sosta riguardo agli approvvigionamenti. I primi si lamentavano con le autorità croate che gli alimenti

\footnotetext{
55 ASMAE, GAB-AP 1923-1943, b. 1512, Approvvigionamento della Dalmazia, 12.8.1941. Per esempio i macellai di Sebenico non potevano portare in Dalmazia i beni comprati in Croazia. ASMAE, GAB-AP 1923-1943, b. 1512, Telegramma di Bozzi a Bastianini, senza data [luglio 1941].

56 ASMAE, GAB-AP 1923-1945, b. 1498, accordo italo-croato sulla soppressione delle linee doganali tra Dalmazia e Croazia, 28.10.1941; AUSSME, DS, b. 543, All. 398, al DS di novembre 1941, Provvedimenti conseguenti all'accordo italo-croato sulla soppressione delle linee doganali, 13.11.1941.

57 VA, NDH, k. 310a, reg. br. 2/24, Zaključci hrvatsko-talijanskog gospodarskog povjereništva o gospodarskim pitanjima na područjima u kojima se obavljaju vojničke operacije, prilog A, Zagreb, 27.10.1941; ASMAE, GAB-AP 1923-1943, b. 1512, Coordinamento e funzionamento dei Servizi dell'Alimentazione per la Dalmazia, Roma, 6.11.1941.

$5^{8}$ Così vennero distribuiti ancora all'inizio di settembre gli alimenti previsti sull'isola di Rab: due chili e mezzo di farina, un po' di pasta, riso, olio, grasso e sapone. Yad Vashem, O 33, Nr. 8367: Tagebuch eines unbekannten Mönches, Eintrag vom 8.9.1943. Questo è particolarmente degno di nota perché la situazione alimentare in Italia era tutt'altro che buona. Cfr. MORGAN, Philip, The Fall of Mussolini. Italy, the Italians and the Second World War, Oxford, Oxford University Press, 2007, pp. 61 et seq.

59 HDA, Ministarstvo vanjskih poslova [Ministero degli affari esteri, MVP], k. 4, T. I. 88, OUP Ministrastvu vanjskih poslova na ruke Min. Dr. Lorkovića 13.3.1943.
} 
non arrivassero con i tempi giusti; i secondi accusavano invece l'esercito italiano di ostacolare i trasporti così come di sequestrare cibo e mezzi di trasporto ${ }^{60}$.

Nel gennaio del 1942 il generale Ambrosio si lamentò con l'intermediario croato perché non si era ancora fatto nulla almeno per impedire che l'approvvigionamento «per motivi politici e umanitari» non peggiorasse ulteriormente ${ }^{61}$. Anche le autorità locali croate scrissero un'infinità di rapporti allarmati ai loro superiori, ma la situazione generale non cambiò affatto ${ }^{62}$.

Ogni tanto, per non dire raramente, si riuscì ad avere una buona collaborazione fra le autorità militari italiane e croate. Così per esempio l'esercito italiano consegnò alle autorità croate, come concordato, determinati alimenti affinché fossero distribuiti fra la popolazione ${ }^{63}$.

A causa della situazione bellica non fu possibile ottenere un considerevole miglioramento della situazione, tuttavia gli italiani posero in essere una politica che consentì loro di ottenere alcuni successi. Così, dopo la distribuzione della farina a Makarska e nei dintorni, i contadini dei paesi vicini portarono di nuovo i loro prodotti al mercato e così la situazione alimentare migliorò un po'. Furono i contadini a elaborare questo sistema perché le autorità locali croate a Makarska non avevano distribuito alimenti razionati - come la farina - che sarebbero spettati a loro, così come al resto della popolazione ${ }^{64}$.

\section{Problema dell'approvvigionamento nell'entroterra croato (seconda e} terza zona)

Le coste dalmate rimaste alla Croazia e l'entroterra dalmata erano le zone che più di altre soffrivano di carenze alimentari. Nell'autunno del 1941 sia le autorità croate sia quelle italiane da punti diversi della seconda zona fecero sapere che alcuni generi alimentari scarseggiavano e che i prezzi stavano salendo vertiginosamente. Della mancanza di generi alimentari soffrivano in modo particolare i serbi visto che appartenevano alla parte più povera della popolazione ${ }^{65}$. Si trattava di persone che in estate si erano rifugiate nelle foreste per sfuggire agli attacchi degli Ustascia e

${ }^{60} \mathrm{HDA}$, OUP, k. 10, Nr. 13065, 2a Armata al Commissariato Generale Amministrativo, Sussa, 24.11.1941; HDA, OUP, k. 28, Nr. 15314, Ministrastvo vanjskih poslova općem upravnom povjereništvu, novembre 1942.

${ }^{61}$ NARA, T-821, R 197, Nr. 888 f., 2. Armata al Commissariato Generale Amministrativo, 10.1.1942.

62 HDA, OUP, k. 17, Nr. 13785, OUP Ministarstvu unutarnjih poslova, 25.5.1942; HDA, OUP, k. 11, br. 13082, Velika Župa Cetina Općem upravnom povjereništvu, Sinj, 22.1.1942.

63 AUSSME, DS, b. 525, All. 39, Lettera al comandante divisione Marche, 17.2.1942; HDA, OUP, k. 3, Nr. 12046, OUP Ministarstvu Unutarnjih Poslova, Sušak, 28.10.1941; NARA, T-821, R. 294, Nr. 78, Il Commissario generale Amministrativo al Comando della 2a Armata, Sussa, 30.7.1943.

${ }^{64}$ AUSSME, DS, b. 881, All. 102, al DS settembre 1942, Relazione mensile sul servizio "P" dal 15 agosto al 15 settembre 1942.

$65 \mathrm{HDA}$, OUP, k. 12, Nr. 13239, Comando della $2^{\circ}$ Armata al Commissariato Generale Amministrativo dello Stato Indipendente di Croazia, 4.12.1941 HDA, OUP, k. 2, Nr. 11854, OUP Državnom Ravnateljstvu za Prehranu NDH, Sušak, 25.9.1941; ASMAE, GAB-AP 1923-1943, b. 1497, Situazione in Erzegovina, 23.10.1941. 
dopo la rioccupazione operata dalla 2a Armata italiana furono inviati con il bando 7 settembre a rientrare nelle loro case ${ }^{66}$. Già nel settembre del 1941 si ebbe notizia di morti per fame. Per alleviare il problema l'esercito italiano distribuì farina alla popolazione addebitandola al $\mathrm{NDH}^{67}$. In particolari occasioni - come il compleanno di Ante Pavelić, per l'entrata in servizio di Mario Robotti come comandante della 2a Armata o quando una personalità visitava una località - si provvedeva in egual modo dalla distribuzione di alimenti68.

Nella primavera del 1943 si verificò una nuova crisi alimentare nell'Erzegovina e sulla costa. Dubrovnik, Mostar e le isole croate furono particolarmente colpite ${ }^{69}$. La situazione fu aggravata dalla devastazione causata dall'operazione Weiß e dalla ritirata dell'esercito italiano da alcune guarnigioni che causò un'ondata di profughi verso la costa. Al governo croato non rimase che chiedere aiuto a Germania e Italia. La Germania inviò mais e patate, ma non rispettò le quantità promesse ${ }^{70}$. In aprile mise a disposizione altre dodicimila tonnellate di cereali e si disse disposta a occuparsi dell'approvvigionamento dell'esercito croato per tenerlo in grado di combattere. Il NDH aveva però bisogno di ulteriori trentamila tonnellate per garantire l'alimentazione della popolazione. Il Reich in questo senso promise «le quantità di cibo essenziali» ${ }^{11}$.

Allo stesso tempo il commissario generale amministrativo croato presso la 2a Armata italiana cercò di trovare un accordo con le autorità militari italiane per l'approvvigionamento delle regioni costiere oltre Trieste e Fiume, così come ulteriori rifornimenti per le città di Ragusa e Almissa $^{72}$. Poiché il Supersloda73 già in marzo aveva inviato sette vagoni di alimenti alle due città e dato che la maggior parte degli alimenti che erano stati messi a disposizione dal 1941 non erano stati pagati dallo Stato croato, le autorità italiane non erano propense a mettere a disposizione nuove derrate alimentari fino a quando non fossero stati saldati i vecchi conti74. Nel complesso gli

\footnotetext{
66 AUSSME, DS, b. 583, rac. 157, Nr. 3, Bando 7 settembre 1941.

67 ASMAE, GAB-AP 1923-1943, b. 1512, Situazione alimentare nella Dalmazia Croata, Zagreb, 5.9.1941; ASMAE, GAB-AP 1923-1943, b. 1504, Telespresso GAB-AP an R. Legazione a Zagabria, 24.9.1941.HDA, OUP, k. 16, Nr. 13708, Corrispondenza tra il Comando della 2a Armata e il Commissariato Generale Amministrativo dello Stato Indipendente di Croazia, gennaio-marzo 1942.

${ }^{68}$ HDA, OUP, k. 41, Nr. 16536, Comando della 2a Armata ai comandi dei CdA, Sussa, 12.6.1943; HDA, OUP, k. 40, Nr. 16381, Comando della 2a Armata, Sussa, 11.5.1943; ORTONA, Egidio, «Diario sul Governo della Dalmazia (1941-1943)», in Storia Contemporanea, 18, 6/1987, p. 1365-1403, p. 1373.

69 ASMAE, GAB-AP 1923-1943, b. 1503, Casertano an GAB-AP, Zagreb, 27.3.1943.

70 ASMAE, GAB-AP 1923-1943, b. 1503, Casertano, an GAB-AP, Zagreb, 28.3.1943.

${ }^{71}$ PA-AA, Büro des Staatssekretärs (StS) Kroatien, Bd. 4, R. 29.668, Nr. 693, Nr. 428f., Auswärtiges Amt (AA) an Botschafter von Rintelen, Berlin, 26.4.1943; BA-MA, RH 31-III/9, Nr. 49f., Vorsprache des Dt. Bev. Generals beim Poglavnik am 20.5.1943.

72 ASMAE, GAB-AP 1923-1943, b. 1503, Verbale della riunione circa il rifornimento dei viveri alle popolazioni croate della costa e dell'interno, 24.3.1943.

73 Il comando della seconda armata italiana venne rinominato nel maggio del 1942 "Comando supremo forze armate Slovenia e Dalmazia", abbreviato in Supersloda, e fu posto direttamente alle dipendenze del Comando supremo.

${ }^{74}$ HDA, MVP, k. 2, Brzojavi iz 1943.,Poslanstvo Nezavisne Države Hrvatske u Rimu Ministarstvu Vanjskih Poslova, Roma, 31.3.1943. Il comando dell'esercito proibì al Supersloda di distribuire altri alimenti fino a
} 
italiani avevano consegnato fino al 30 giugno 1943 1665,2 tonnellate di farina, 171,7 di pasta, 143,7 di riso e 17,6 di carne, limitandoci agli alimenti più importanti. Di questi i croati furono in grado di rimborsare 16,7 tonnellate di farina, 12 di farina di mais, 0,4 di pasta, 0,5 di riso e 0,4 di carne 75 . Tuttavia per ragioni politiche e sulla base di considerazioni di carattere militare la 2a Armata distribuì altri alimenti. Al 24 aprile 1943 si arrivò a 340 tonnellate di farina di cui solo per Dubrovnik 150. L'ambasciatore croato ringraziò a fine luglio per l'aiuto che la 2a Armata aveva prestato alla popolazione indigente dopo un'operazione militare nella zona del Biokovo ${ }^{76}$. Il generale Robotti volle comunque indicazioni dai suoi superiori sui comportamenti che avrebbe dovuto tenere in futuro sulla base di motivazioni politico-militari77.

Un ulteriore problema era rappresentato dai profughi che si erano spostati, al seguito dell'esercito italiano, verso la costa. Lo Stato croato si occupava di loro in modo insufficiente. Nonostante il divieto imposto al Supersloda di distribuire ulteriori alimenti fu di nuovo l'esercito italiano a farsi carico del loro sostentamento. In questo modo il prestigio italiano, la calma e l'ordine, così come condizioni sanitarie dignitose, sarebbero state mantenute. Mentre le guarnigioni italiane distribuivano gli alimenti più necessari si fece pressione sul governo croato affinché si facesse carico direttamente delle esigenze dei profughi ${ }^{78}$.

Nelle città di Kraljevica e Crikvenica le razioni mensili erogate dalle autorità croate consistevano in 15 chili di patate, un chilo e mezzo di verdure, tre chili di mais, seicento grammi di pasta, un chilo di farina, un quarto di litro d'olio. Le autorità militari italiane definirono queste quantità come palesemente insufficienti e vollero integrarle almeno con zucchero, pomodori in scatola e formaggio79.

I militari italiani utilizzarono la situazione degli approvvigionamenti anche per accattivarsi le simpatie della popolazione, soprattutto a Dubrovnik e sulle isole, in modo da ottenere una posizione favorevole per futuri aggiustamenti di confine. A Dubrovnik nel 1942 gli italiani fecero una forte propaganda che estesero anche ai paesi vicini. A questo scopo venne aperta una scuola elementare e un liceo in cui distribuivano gratuitamente cibo e vestiti. Alcuni rapporti delle

quando i croati non avessero pagato i loro debiti. ASMAE, GAB-AP 1923-1943, b. 1503, Ufficio collegamento II. Armata an GAB-AP, 17.4.1943; ASMAE, GAB-AP 1923-1943, b. 1503, Ufficio collegamento II. Armata an GAB-AP, 26.3.1943.

${ }^{75}$ ASMAE, GAB-AP 1923-1943, b. 1503, Totale generale delle cessioni, restituzioni e rimanenze da parte delle autorità civili e militari croate dall'inizio fino al 30 giugno 1943.

${ }^{76}$ NARA, T-821, R. 294, Nr. 78, OUP al Comando della 2a Armata, Sussa, 30.7.1943.

77 TALPO, Oddone, op. cit., vol. 3, Situazione alimentare in Croazia, 24.4.1943, pp. 553-555.

${ }^{78}$ HDA, OUP, k. 41, Nr. 16540, 16550, Corrispondenza tra il Comando della 2. Armata e il Commissariato Civile Croato, Sussa, 18./20.6.1943; HDA, OUP k. 36, Nr. 16021, Sinj, Juni 1943.ASMAE, GAB-AP 1923-1943, b. 1503, Castellani al GAB-AP, 23.5.1943; HDA, OUP, k. 41, Nr. 16550, Comando 2a Armata al Commissariato Generale Croato, Sussa, 20.6.1943; HDA, OUP k. 38, Nr. 16259, Comando Supersloda al Commisariato Generale Croato, Sušak, 7.4.1942.

${ }^{79}$ ASMAE, GAB-AP 1923-1943, b. 1503, Ufficio di collegamento con il comando della 2a Armata al GAB-AP, Ufficio Croazia, 7.5.1943. 
autorità croate fecero presente che le misure erano popolari tra la parte più povera della popolazione. Secondo il rapporto fino a quel momento già millesettecento persone avevano fatto richiesta di entrare in territorio italiano ${ }^{80}$.

L'incapacità del NDH di fare fronte all'approvvigionamento per le isole di Brač e Hvar che si trovano di fronte a Spalato fu utilizzata per lo stesso scopo. Dopo che all'isola vennero messi a disposizione alimenti dal consorzio cooperativo di Spalato, si accumularono voci che sostenevano la necessità di chiedere un'annessione all'Italia. Si cercava di suscitare questo stato d'animo continuando ad approvvigionare l'isola prendendo come contropartita ciò che essa poteva offrire (soprattutto olio, olive e legno). Allo stesso tempo si cercava di privare di potere in misura crescente i funzionari croati: ciò avrebbe sostenuto la tendenza autonomista su entrambe le isole. $\mathrm{Su}$ altre isole si svilupparono simili dinamiche. Il governatore della Dalmazia organizzò uno scambio di beni con l'isola di Pag che avrebbe dovuto migliorare la situazione degli approvvigionamenti alimentari e «le relazioni tradizionali con Zara». A Pag si giunse a una guerra aperta fra gli abitanti per decidere a chi dovesse appartenere l'isola. A seguito della richiesta di cento capifamiglia e di alcune madri arrabbiate, insieme ai loro figli, di annessione all'Italia si tenne una manifestazione di circa duecento Ustascia per la permanenza in Croazia ${ }^{81}$.

\section{Questione degli approvvigionamenti e lotta ai partigiani}

Già altri eserciti avevano usato la leva dei rifornimenti come arma nella lotta contro gli insorti. Se questo elemento non può quindi essere considerato come una peculiarità del tentativo italiano di costruzione di un impero, tuttavia deve essere incluso nel quadro. Tutti coloro che non accettavano il dominio italiano e che o si erano uniti ai partigiani o li "sostenevano", almeno agli occhi degli italiani - per quanto questo "sostegno" potesse essere giustificato a piacimento dall'esercito italiano e dalle autorità locali - vennero puniti anche con la sottrazione di cibo. Coloro che, al contrario, si comportavano in modo collaborativo o quantomeno neutrale, venivano premiati anche con approvvigionamenti alimentari.

La fame non si limitava a demoralizzare la popolazione, ma la spingeva fra le braccia degli insorti comunisti. Le autorità locali croate fecero notare che una tale situazione poteva essere

\footnotetext{
80 VA, NDH, k. 320, reg. br. 2/10-99, Ministarstvo unutarnjih poslova Ministarstvu vanjskih poslova, Zagreb, 8.10.1942.

81 NARA, T 821, R 401, Nr. 232-235, Il Governatore della Dalmazia, Giuseppe Bastianini, al Generale Mario Roatta, Comandante Superiore FF.AA. Slovenia-Dalmazia, Zara, 3.11.1942; T 821, R 401, Nr. $244 f$., Il Governatore della Dalmazia al Comandante della 2a Armata, Zara, 22.4.1942; T 821, R 401, Nr. 251 f., 258.
} 
facilmente sfruttata dalla propaganda nemica. La popolazione avrebbe dato la colpa della miseria allo Stato croato e sarebbe stata quindi molto più sensibile alla propaganda dei partigiani ${ }^{82}$.

Quando i partigiani iniziarono a proclamare di voler trattare bene la popolazione e di volersi occupare del suo sostentamento nelle aree sotto il loro controllo, gli occupanti furono costretti a fare lo stesso per evitare che sempre più persone passassero dalla loro parte ${ }^{83}$. Anche gli occupanti dovettero riconoscere che perfino la migliore propaganda falliva quando «fame e stenti iniziavano a colpire la vita del popolo». La Wehrmacht tuttavia si rese conto che «l'energico sostegno e aiuto alla popolazione» avrebbe rappresentato la miglior forma di propaganda solamente nell'estate del $1943^{84}$. Fino alla capitolazione dell'Italia la Wehrmacht si trovava a occupare principalmente le regioni croate più floride dal punto di vista alimentare dove il problema era meno sentito. Inoltre le autorità tedesche per rispetto della formale sovranità della Croazia (presa a cuore in particolare dal Ministero degli Esteri) non avevano né effettuato propaganda né si erano occupate del sostentamento della popolazione ${ }^{85}$. Entrambe queste decisioni vennero modificate a partire dal 1943 anche se la propaganda tedesca riguardava essenzialmente la lotta al mercato nero e in generale allo sforzo di aumentare e mettere in sicurezza i raccolti e non menzionava spesso aiuti materiali ${ }^{86}$.

Per gli italiani al contrario nell'economia della lotta ai partigiani la distribuzione di cibo ebbe un ruolo relativamente grande. L'obiettivo era tenere la popolazione lontana dai comunisti grazie a un'equa distribuzione del cibo alla popolazione. Queste misure, in sé del tutto positive per la popolazione, vennero tuttavia affiancate dall'esercito italiano a una metodologia criminale di lotta ai ribelli.

I paesi o le regioni che venivano identificate come "comuniste" sulla base di un assalto che era avvenuto da quelle parti o perché da un certo luogo alcune persone si erano rifugiate nelle foreste, venivano escluse dai rifornimenti. Così un villaggio non ricevette più alimenti dopo che $\mathrm{i}$ partigiani avevano ucciso una famiglia filo-italiana ${ }^{87}$. Dopo che il prefetto della provincia di Zara, Vezio Orazi, venne ucciso il 26 maggio 1942 in un agguato, Bastianini ordinò la sospensione della

\footnotetext{
82 HDA, OUP, k. 14, Nr. 13464, Brzojav lokalnih vlasti u Trebinju Ministrarstvu Unutarnjih Poslova, marzo 1942; HDA, OUP, k. 11, Nr. 13082, Velika Župa Cetina Općem upravnom povjereništvu, Sinj, 22.1.1942.

83 AUSSME, DS, b. 646, All. 34, al DS Marzo 1942, Notiziario Nr. 21, 10.3.1942.

84 BArch, RH 26-114/28, Propagandabericht vom 25.11.1943.

85 Il Reich a partire dal 1943 mise a disposizione quantità di cibo. SUNDHAUSSEN, Holm, Wirtschaftsgeschichte Kroatiens, cit., p. 266. In questo modo il Reich rafforzò il regime degli Ustascia che distribuì gli alimenti per perseguire i suoi obiettivi politici. Qui si possono ravvisare di nuovo le differenze fondamentali nei piani e negli atteggiamenti del Reich tedesco e dell'Italia nei confronti del NDH. Un quadro simile si presenta anche nella Grecia occupata: FONZI, Paolo, «Regimes of Supply in Greece 19411944», presentato al convegno, The Second World War in Southeastern Europe, Berlin, 4-6 ottobre 2017.

86 BArch, RH 26-114/21, 114. I.D., Propagandabericht, 24.6.1943.

87 AJ, Fond 110, k. 736, Nr. $177 f$.
} 
distribuzione di cibo per i villaggi che si trovavano nelle vicinanze del luogo dell'agguato ${ }^{88}$. Quando a Brač nell'aprile del 1942 vennero rapiti un tenente e tre ragazze dai partigiani e il primo, nonostante un ultimatum, non venne liberato il comandante della divisione "Bergamo" ordinò di uccidere quattro ostaggi come ritorsione e prese delle misure anche in relazione all'invio di alimenti: «Proprio oggi avevo deciso di mettere a disposizione dei più bisognosi una grossa quantità di riso e di farina, una concessione che naturalmente ora ho ritirato» ${ }^{89}$. A volte però si fecero anche altre indagini per non interrompere la fornitura di cibo alla parte "sana" (secondo la prospettiva italiana) della popolazione ${ }^{90}$. Le persone che collaboravano con gli italiani (per esempio aiutando i soldati italiani che scappavano dai partigiani) venivano privilegiate nella distribuzione degli alimenti91 . Ciò provocò ripetutamente proteste croate contro le distribuzioni di cibo ai serbi: secondo loro in questo modo gli alimenti avrebbero trovato direttamente la loro strada verso i partigiani. Tuttavia i comandanti italiani non accettarono una simile intromissione nei loro affari ${ }^{2}$.

Nella propaganda per i soldati che - come ha argomentato in modo convincente Amedeo Osti Guerrazzi - si trovava in un dilemma insolubile fra il discredito di tutti gli slavi e la discriminazione fra la «popolazione amica» e i «banditi»93, si fece sempre più ricorso alla missione civilizzatrice dell'Italia. Per esempio su «La tradotta» del 29 novembre 1942 venne pubblicata una foto che mostrava un soldato nell'atto di porgere un pezzo di pane a un ragazzo piccolo e gracile. La foto venne corredata da un breve articolo dal titolo «Latinità» in cui da una parte veniva idealizzata la generosità dei soldati italiani di fronte alla popolazione e dall'altra si metteva in guardia dai «serpenti» comunisti. L'articolo si concludeva con: «Ma siamo sufficientemente grandi e civili e forti nelle armi per sfamare un fanciullo innocente, e distruggere senza alcuna pietà e sino all'ultimo chi si mostri nemico di Roma»94.

Questa percezione che l'esercito italiano aveva di sé includeva entrambi i pilastri della costruzione imperiale: la forza militare così come la superiorità civilizzatrice.

I rifornimenti sottoposti a regolazioni portarono sì dei risultati, ma non riuscirono a impedire la resistenza alla dominazione italiana. La repressione dei militari italiani nei confronti della popolazione civile che andava dalla distruzione di interi paesi fino all'esecuzione di ostaggi pesava

\footnotetext{
88 Ordinanza di Bastianini del 7 giugno 1942, Nr. 453, pubblicato in EGIĆ, Obrad, Narodnooslobodilački partizanski odred za sjevernu Dalmaciju, Zadar, Narodni list, 1987, pp. 247 et seq.

89 NARA, T-821, R. 299, Nr. 334-338, Il rapporto del Comandante della divisione «"Bergamo"», aprile 1943.

$9^{\circ}$ HDA, OUP, k. 38, Nr. 16220, Corrispondenza tra l'OUP e 2a Armata, Sussak, aprile 1943.

91 NARA, T-821, R. 418, Nr. 310-313, VI CdA, Relazione sull'attività di contropropaganda per il mese di luglio 1943.

92 HDA, OUP, k. 25, Nr. 14823, Corrispondenza tra le autorità locali di Ravno e OUP, April 1942;

93 OSTI GUERRAZZI, Amedeo, The Italian Army in Slovenia. Strategies of Antipartisan Repression 1941-1943, New York, Palgrave Macmillan, 2013.

94 NAW, T-821, R. 414, Nr. 222, “Latinità” in «La tradotta» Nr. 26/I, 29.11.1942.
} 
più di qualunque altra misura positiva. I saccheggi operati dai soldati italiani ebbero le stesse conseguenze. Quando nell'agosto del 1943 si tornò a chiedere all'Italia di occuparsi dei rifornimenti delle zone costiere il dipartimento per la Croazia del Ministero per gli Affari Esteri mise in chiaro che, nonostante il pagamento da parte dei croati fosse incerto, era necessario farsene carico. In questo modo si tentò di operare una sorta di risarcimento per le distruzioni operate in precedenza95. Se effettivamente dal punto di vista dei danni materiali queste misure potevano in parte migliorare la situazione le vite umane falciate dalla guerra contro i partigiani non erano risarcibili.

\section{Conclusione}

L'Italia fascista ha cercato di costruire un impero che potesse fondarsi sulla civilizzazione romana. Con l'esempio dei rifornimenti alimentari allo Stato indipendente di Croazia è stato qui mostrato come le autorità italiane, principalmente militari, si siano poste di fronte al problema. L'argomento centrale si basa sull'ipotesi che esse fossero influenzate da un idealtipo fascista dell'Impero romano. In questo senso hanno giocato un ruolo sia la spietatezza dimostrata nei confronti dei nemici, sia l'offerta di inclusione offerta alla popolazione che avrebbe dovuto essere integrata nell'Impero. Quando le autorità italiane furono messe concretamente di fronte alla disastrosa situazione alimentare si resero conto che era giunto il momento di attaccare per difendere il "prestigio" italiano, per legittimare le rivendicazioni su queste aree e per mettere in pratica la rappresentazione "imperiale" del loro modo di agire.

Nelle zone annesse e occupate dello Stato indipendente di Croazia la politica alimentare dell'esercito italiano aveva alcuni obiettivi principali. In prima battuta si desiderava portare avanti una politica anticroata il cui scopo era far sì che determinati territori finissero saldamente in mani italiane. Inoltre la popolazione doveva essere convinta della bontà dell'idea di un impero fascista italiano e dei vantaggi (migliori approvvigionamenti medici, progetti di infrastrutture e migliori rifornimenti) che avrebbe comportato il farne parte, mentre i tedeschi nel loro impero dell'Est europeo lasciavano morire di fame la popolazione ${ }^{96}$. I fascisti videro gli aiuti alimentari come un modo per influenzare a piacimento la popolazione e per portare avanti sia una strategia a breve termine sia obiettivi imperiali nel lungo periodo ${ }^{97}$. I militari italiani capirono anche prima

\footnotetext{
95 ASMAE, GAB-AP 1923-1943, b. 1503, Appunto, Roma, 19.8.1943.

96 DIECKMANN, Christoph, QUINKERT, Babette (herausgegeben von), Kriegführung und Hunger 1939-1945. Zum Verhältnis von militärischen, wirtschaftlichen und politischen Interessen, Göttingen, Wallstein Verlag, 2015; GERLACH, Christian, Kalkulierte Morde. Die deutsche Wirtschafts- und Vernichtungspolitik in Weißrußland 1941 bis 1944, Hamburg, Hamburger Edition, 2000.

${ }^{97}$ Considerazioni analoghe per la Grecia occupata dall'Italia sono state presentate da Paolo Fonzi. Le élites italiane erano convinte che anche l'inclusione del paese ellenico in un impero italiano sarebbe passato
} 
dei loro colleghi tedeschi che un trattamento generalmente corretto della popolazione civile, di cui l'approvvigionamento alimentare era parte determinante, poteva essere un'arma nella lotta contro i ribelli.

Tuttavia questa strategia non ebbe successo perché questi benefici vennero vanificati al contempo nella lotta militare contro i partigiani. L'esercito italiano adottò gli stessi sistemi di lotta dei loro alleati di cui fecero le spese le stesse popolazioni di cui ci si voleva accattivare le simpatie.

attraverso la loro capacità di fornire un approvigionamento alimentare alla popolazione civile greca. FONZI, Paolo, op. cit. 


\section{L'AUTORE}

Sanela SCHMID ha conseguito il dottorato presso l'università di Berna con una tesi dal titolo L'occupazione tedesca $e$ italiana nello Stato Indipendente di Croazia 1941-1943/45. Ha successivamente lavorato al quattordicesimo volume dell'edizione di documenti Die Verfolgung und Ermordung der europäischen Juden durch das nationalsozialistische Deutschland 1933-1945 [La persecuzione e l'uccisione degli ebrei europei per mano della Germania nazionalsocialista 1933-1945] intitolato Südosteuropa und Italien (München, De Gruyter Oldenbourg, 2017) di cui ha anche curato l'introduzione alla sezione jugoslava. I suoi principali interessi di ricerca si concentrano sulla storia della Seconda guerra mondiale in Jugoslavia e sulla storia della Shoah. È inoltre autrice di: Zwangsarbeit im italienischen Bereich, in HODZIC, Sanela, SCHÖLZEL, Christian, Zwangsarbeit im "Unabhängigen Staat Kroatien" 1941-1945, Berlin, LIT Verlag, 2013, pp. 136-174; «"Italiani brava gente?". Storiografia recente dell'occupazione italiana in Croazia durante la seconda guerra mondiale», in Ventunesimo Secolo. Rivista di Studi sulle Transizioni, 7, 16, 2/2008, pp. 31-55.

URL: < http://www.studistorici.com/progett/autori/\#Schmid > 